\title{
Reversible Biholomorphic Germs
}

\author{
Patrick Ahern \\ Mathematics Department, University of Wisconsin \\ Madison, WI 53706, USA \\ e-mail: ahern@math.wisc.edu \\ and \\ Anthony G. O'Farrell ${ }^{1}$ \\ Mathematics Department, National University of Ireland \\ Maynooth, Co. Kildare, Ireland \\ e-mail: anthonyg.ofarrell@gmail.com
}

December 8, 2008

\section{Abstract}

Let $G$ be a group. We say that an element $f \in G$ is reversible in $G$ if it is conjugate to its inverse, i.e. there exists $g \in G$ such that $g^{-1} f g=f^{-1}$. We denote the set of reversible elements by $R(G)$. For $f \in G$, we denote by $R_{f}(G)$ the set (possibly empty) of reversers of $f$, i.e. the set of $g \in G$ such that $g^{-1} f g=f^{-1}$. We characterise the elements of $R(G)$ and describe each $R_{f}(G)$, where $G$ is the the group of biholomorphic germs in one complex variable. That is, we determine all solutions to the equation $f \circ g \circ f=g$, in which $f$ and $g$ are holomorphic functions on some neighbourhood of the origin, with $f(0)=g(0)=0$ and $f^{\prime}(0) \neq 0 \neq g^{\prime}(0)$.

\section{Introduction}

\subsection{General Setting}

Let $G$ be a group. We say that an element $f \in G$ is reversible in $G$ if it is conjugate to its inverse, i.e. there exists $g \in G$ such that $g^{-1} f g=f^{-1}$. We

\footnotetext{
${ }^{1}$ Supported by Grant SFI RFP05/MAT0003 and the ESF Network HCAA.

${ }^{2}$ Mathematics Subject Classification 2000: 30D05, 39B32, 37F99, 30C35.
} 
denote the set of reversible elements by $R(G)$. For $f \in G$, we denote by $R_{f}(G)$ the set (possibly empty) of reversers of $f$, i.e. the set of $g \in G$ such that $g^{-1} f g=f^{-1}$.

The set $R(G)$ always includes the set $I(G)$ of involutions (elements of order at most 2). Indeed, it also includes the larger set

$$
I^{2}(G)=\left\{\tau_{1} \tau_{2}: \tau_{i} \in I(G)\right\}
$$

of strongly-reversible elements, i.e. elements that are reversed by an involution. If $g \in G$ reverses $f \in G$, then $g^{2}$ commutes with $f$, i.e. $g^{2}$ belongs to the centraliser $C_{f}(G)$. More generally, the composition of any two elements of $R_{f}(G)$ belongs to $C_{f}(G)$. For this reason, an understanding of centralisers in $G$ is a prerequisite for an understanding of reversers.

The following easily-proved theorem characterises the reversers of an element, in any group.

Theorem 1.1 (Basic Theorem). Let $G$ be a group and $f, g \in G$. Then the following three conditions are equivalent:

1. $g \in R_{f}(G)$;

2. there exists $h \in G$ with $g^{2}=h^{2}$ and $f=g^{-1} h$;

3. there exist $h \in G$ such that $f=g h$ and $f^{-1}=h g$.

This then yields two characterisations of reversibility:

Corollary 1.2. Let $G$ be a group and $f \in G$. Then the following three conditions are equivalent:

1. $f \in R(G)$;

2. there exist $g, h \in G$ with $g^{2}=h^{2}$ and $f=g^{-1} h$;

3. there exist $g, h \in G$ such that $f=g h$ and $f^{-1}=h g$.

This shows that reversibility is interesting only in nonabelian groups in which there are elements with multiple square roots. In any specific group, it is interesting to give more explicit characterisations of reversibility than those of this theorem. 
This paper is about the reversible elements in the group of invertible biholomorphic germs and some of its subgroups.

We shall characterise these elements, and their reversers, and the stronglyreversible elements, in explicit ways. We shall also consider some related questions.

The theory of reversibility for formal power series in one variable has already been dealt with in [10]. We shall see (cf. Section 4) that there exist germs $f \in G$ that are formally reversible, but not holomorphically reversible.

\subsection{Our specific groups}

For the remainder of the paper, we shall denote by $G$ the group of biholomorphic germs at 0 in one complex variable. Thus an element of $G$ is represented by some function $f$, holomorphic on some neighbourhood (depending on $f$ ) of 0 , with $f^{\prime}(0) \neq 0$, and two such functions represent the same germ if they agree on some neighbourhood of 0 . The group operation is composition. The identity is the germ of the identity function 1.

The multiplier map $m: f \rightarrow m(f)=f^{\prime}(0)$ is a homomorphism from $G$ onto the multiplicative group $\mathbb{C}^{\times}$of the complex field.

Obviously, since $\mathbb{C}^{\times}$is abelian, the value $m(f)$ depends only on the conjugacy class of $f$ in $G$.

We denote

$$
\begin{aligned}
H & =\{f \in G: m(f)=\exp (i \pi q), \text { for some } q \in \mathbb{Q}\} \\
H_{0} & =\{f \in G: m(f)= \pm 1\}=\operatorname{ker} m^{2},
\end{aligned}
$$

and

$$
G_{1}=\operatorname{ker} m \text {. }
$$

These normal subgroups have $G_{1} \leq H_{0} \leq H \leq G$.

Further, for $p \in \mathbb{N}$, we define

$$
G_{p}=\left\{f \in G_{1}: f^{(k)}(0)=0 \text { whenever } 2 \leq k \leq p\right\},
$$

and

$$
A_{p}=G_{p} \sim G_{p+1} .
$$

Then $G_{1}$ is the disjoint union of $\{1\}$ and the sets $A_{p}$. For $f \in G_{1}$, with $f \neq 1$, we denote by $p(f)$ the unique $p$ such that $f \in A_{p}$. The natural number $p(f)$ is a conjugacy invariant of $f$ (with respect to conjugation in $G$ ), so that each $G_{p}$ is a normal subgroup of $G$.

For $f \in G_{p}$, we may write $f(z)=z+f_{p+1} z^{p+1}+O\left(z^{p+2}\right)$. The map $f \mapsto f_{p+1}$ is a group homomorphism from $G_{p}$ onto $(\mathbb{C},+)$. Thus $f_{p+1}$ is a conjugacy invariant of $f$ in $G_{p}$. It is even invariant under conjugation in $G_{1}$, but it is not invariant 
under conjugation in $G$. Each $f \in A_{p}$ may be conjugated to the form $g^{-1} f g=$ $z+z^{p+1}+a(f) z^{2 p+1}+O\left(z^{2 p+2}\right)$, and then the complex number $a(f)$ is a conjugacy invariant of $f$ in $G$.

The invariants $p(f)$ and $a(f)$ classify the elements of $G_{1} \sim\{1\}$ up to formal conjugacy. The complete biholomorphic conjugacy classification requires additional invariants, and these have been provided by the equivalence class of the EV data $\Phi(f)$ of Écalle-Voronin theory, which is reviewed briefly in Section 2 below.

For $f \in H_{0} \sim G_{1}$, a complete set of conjugacy invariants (with respect to conjugacy in $G$ ) is provided by $m(f)=-1$ and the conjugacy class of $f^{2}$, which belongs to $G_{1}$. (See Theorem 2.3 below.)

\subsection{Summary of results}

It is obvious that each group homomorphism maps the reversible elements of its domain to reversible elements of its target, and that the only reversible elements in an abelian group are its involutions. Hence $R(G) \subset H_{0}$. Consequently, the reversible elements in all subgroups of $G$ lie in $H_{0}$.

Also, it is always true that for $f \in G_{1}, p(f)=p\left(f^{-1}\right)$. Also, by purely formal considerations [10], the condition $a(f)=a\left(f^{-1}\right)$ is equivalent to $a(f)=(p(f)+$ $1) / 2$. Thus the short answer to the question of which $f \in G$ are reversible in $G$ is the following:

Proposition 1.3. Let $f \in G$. Then $f \in R(G)$ if and only if (exactly) one of the following holds:

1. $f^{\prime}(0)=1$, and $\Phi(f)$ is equivalent to $\Phi\left(f^{-1}\right)$;

2. $f^{\prime}(0)=-1$, and $f^{2} \in R(G)$.

For Part 2, see Corollary 4.1. However, we can provide much more explicit information about reversibility in $G$.

In general groups, a reversible element $f$ may have no reversers of finite order. If there is a reverser of finite order, then there is one whose order is a positive power of 2. Only involutions can have a reverser of odd order. In our present group $G$, we have the following:

Theorem 1.4. Let $f \in A_{p}$, for some $p \in \mathbb{N}$, and $g \in R_{f}(G)$. Then $g$ has finite even order $2 s$, for some $s \in \mathbb{N}$ with $p / s$ an odd integer.

We shall give examples (cf. Section 4) to show that there are $f \in G$ for which the lowest order of a reverser is any preassigned power of 2 .

We can be rather more precise about the order of reversers, but we have to distinguish between "flowable" and "non-flowable" reversible germs $f$. 
Definition. By a flow in $G_{1}$ we mean a continuous group homomorphism $t \mapsto f_{t}$ from $\left(\mathbb{R},+\right.$ ) (a real flow) or $\left(\mathbb{C},+\right.$ ) (a complex flow) into $G_{1}$.

A germ $f \in G_{1}$ is called flowable if and only if there exists a flow $\left(f_{t}\right)$ with $f_{1}=f$. The more precise result about reversers involves technical parameters that are associated to a reversible germ $f \in G_{1}$, and we shall give the statement and proof later (cf. Section 5), after we have explained these parameters.

Theorem 1.5. let $f \in A_{p}$, for some $p \in \mathbb{N}$. Then $f \in R(G)$ if and only if it may be written as $g^{-1} h$, where $g, h \in H$ are germs of finite even order $2 s, g^{2}=h^{2}$, $s \mid p$, and $p / s$ is odd.

As is well-known, each germ of finite order in $G$ is conjugate in $H$ to a rotation through a rational multiple of $\pi$ radians. Indeed an elements $g \in G$ of finite order $\delta$ must have multiplier $\beta=m(g)$ a $\delta$-th root of unity, and is conjugate in $H$ to $z \mapsto \beta z$; in fact the function

$$
\frac{1}{\delta}\left(z+\frac{g(z)}{\beta}+\cdots+\frac{g^{\delta-1}(z)}{\beta^{\delta-1}}\right)
$$

provides a conjugation.

Theorem 1.6. Let $f \in A_{p}$, for some $p \in \mathbb{N}$. Then $f \in R(G)$ if and only if there exists $\psi \in H$ such that

$$
\left(\psi^{-1} f \psi\right)(z)=z+z^{p+1}+\sum_{k=1}^{\infty} c_{k} z^{s k+p+1},
$$

where $p / s$ is an odd integer, and

$$
\left(\psi^{-1} f^{-1} \psi\right)(z)=z-z^{p+1}+\sum_{k=1}^{\infty}(-1)^{k} c_{k} z^{s k+p+1} .
$$

(In other words, $f_{1}=\psi^{-1} f \psi$ is reversed by $z \mapsto \exp (\pi i / s) z$.)

We shall give examples (cf. Section 4) to show that each $p \in \mathbb{N}$ and each $s \mid p$ with $p / s$ odd may occur.

These results allow us to understand reversibility in $G$ : One reverses a germ $f$ essentially by "rotating" it (using a rotation modulo conjugacy), so as to swap the attracting and repelling petals of its Leau flower.

We note some consequences:

Corollary 1.7. Let $f \in G$. Then $f \in R(G)$ if and only if $f^{2} \in R(G)$.

The strongly-reversible elements of $G$ were already identified (in terms of EV data) in [5], but we note the result, which follows immediately from Theorem 1.4 above: 
Corollary 1.8. Let $f \in G$. Then $f \in I^{2}(G)$ if and only if $f \in R(G)$ and one of the following holds:

1. $f \in I(G)$, or

2. $f \in A_{p}$ with $p$ odd.

We note that the case $p=1$ was already given by Voronin [13].

The following summarises our conclusions about reversibility in all the abovenamed subgroups of $G$ :

Corollary 1.9. For each $p \in \mathbb{N}$, we have

$$
(1)=R\left(G_{p}\right)=R\left(G_{1}\right) \subset R\left(H_{0}\right)=I^{2}(G) \subset R(H)=R(G) \subset H_{0},
$$

and the three inclusions are proper.

\section{Conjugacy}

Definition. Let $p \in \mathbb{N}$. Let $\mathfrak{S}$ denote the set of all functions $h$ that are defined and holomorphic on some upper half-plane (depending on $h$ ), and are such that $h(\zeta)-\zeta$ is bounded and has period 1. By Écalle-Voronin p-data (or just EV data) we mean an ordered $2 p$-tuple $\Phi=\left(\Phi_{1}, \ldots, \Phi_{2 p}\right)$, where $\Phi_{1}(\zeta),-\Phi_{2}(-\zeta), \Phi_{3}(\zeta)$, $\ldots,-\Phi_{2 p}(-\zeta) \in \mathfrak{S}$.

Given EV $p$-data $\Phi$ and $q$-data $\Psi$, we say that they are equivalent if $p=q$ and there exist $k \in \mathbb{Z}$ and complex constants $c_{1}, \ldots, c_{2 p}$, such that for each $j$ we have

$$
\Phi_{j+2 k}\left(\zeta+c_{j}\right)=\Psi_{j}(\zeta)+c_{j+1}
$$

(where we define $\Phi_{j}, \Psi_{j}$ and $c_{j}$ for all $j \in \mathbb{Z}$ by making them periodic in $j$, with period $2 p$ ).

Let $f \in G_{1}$. Let $p=p(f)$. Voronin [12] described how to associate Écalle-Voronin data $\Phi(f)=\left(\Phi_{1}, \ldots, \Phi_{2 p}\right)$ to $f$. We shall not recapitulate the construction here ${ }^{1}$, but roughly speaking the $\Phi_{j}$ are obtained as (analytic extensions of) compositions $F_{j} \circ F_{j+1}^{-1}$, where the $F_{j}$ are conformal maps of alternately attracting and repelling Leau petals for $f$, which conjugate $f$ on the petals to translation by 1 near $\infty$. Essentially the same construction was discovered independently by Écalle [9]. They proved the following:

Theorem 2.1 (Conjugacy). Let $f, g \in G_{1}$. Then $f$ is conjugate to $g$ in $G$ if and only if $\Phi(f)$ is equivalent to $\Phi(g)$.

\footnotetext{
${ }^{1}$ For a detailed description, see Voronin's paper [12] or (for full details when $p>1$ ) [5, pp.7-19]. The case $p>1$ was first fully elaborated by Yu. S. Ilyashenko [6].
} 
Theorem 2.2 (Realization). Given any $E V$ data $\Phi$, there exists a function $f \in$ $G_{1}$ having equivalent $E V$ data.

For $f \in H$, the expositions in print usually say that the conjugacy classification is easily reduced to the case of multiplier 1 . We need to consider multiplier -1 , so we need a precise statement. The result goes back to Muckenhoupt [7, Theorem 8.7.6, p. 359].

Theorem 2.3 (Muckenhoupt). Suppose that $f, g \in H$ both have the same multipier $\lambda$, a primitive $s$-th root of unity, where $s \in \mathbb{N}$. Then $f$ and $g$ are conjugate in $G$ if and only if $f^{s}$ and $g^{s}$ are conjugate in $G$.

We supply a proof, partly for the reader's convenience, but also because we wish to draw a useful corollary from it.

Proof. It is evident that if $h^{-1} f h=g$, then $h^{-1} f^{s} h=g^{s}$.

For the other direction, suppose that there exists $h \in G$ with $h^{-1} f^{s} h=g^{s}$.

We have $\left(h^{-1} f h\right)^{s}=g^{s}$, and $m\left(h^{-1} f h\right)=\lambda$. So it suffices to show that

$$
\left\{\begin{aligned}
m(f) & =m(g)=\lambda \\
f^{s} & =g^{s}
\end{aligned}\right\} \Rightarrow f \text { is conjugate to } g .
$$

Let $k=f^{s}$. Then $k \in G_{1}$.

If $k$ is the identity, then $f$ and $g$ are periodic with the same multiplier, so they are conjugate.

If $k$ is not the identity, then the centraliser of $k$ is abelian (see Theorems 3.1 and 3.2 below). Since $f$ and $g$ belong to it, they commute with each other, hence $\left(f^{-1} g\right)^{s}=f^{-s} g^{s}=1$. But $f^{-1} g \in G_{1}$, so $f^{-1} g=1$, and $f$ is actually equal to $g$.

Corollary 2.4. If $f, g \in H$ have as multiplier the same $n$-th root of unity, and $f^{n} \neq 1$, then each $h \in G$ that conjugates $f^{n}$ to $g^{n}$ will also conjugate $f$ to $g$.

\section{Centralisers}

The facts about $C_{f}(G)$, for $f \in G_{1}$, were established by Baker and Liverpool $[1,2,3,4]$ (see also Szekeres [11]).

We may summarise the facts about centralisers as follows:

Theorem 3.1. Suppose that $p \in \mathbb{N}$ and $f \in A_{p}$ is flowable. Then $C_{f}(G)$ is an abelian group, equal to the inner direct product

$$
\left\{f_{t}: t \in \mathbb{C}\right\} \times\left\{\omega^{j}: 0 \leq j \leq p\right\}
$$

where $\left(f_{t}\right)_{t \in \mathbb{C}}$ is a complex flow, and $\omega \in H$ has finite order $p$. 
It follows from Theorem 3.1 that if $f \in G_{1}$ is flowable then $C_{f}\left(G_{1}\right)$ is the flow $\left(f_{t}\right)_{t \in \mathbb{C}}$. It is a remarkable result of Baker and Liverpool that in the non-flowable case $C_{f}\left(G_{1}\right)$ is an abelian group with a single generator $g$. Since $f \in C_{f}\left(G_{1}\right)$ we have $f=g^{d}$ for some integer $d$ (which we can assume to be positive (by replacing $g$ by $g^{-1}$ if necessary). This $g$, which is unique, is usually denoted by $f^{\frac{1}{d}}$.

Theorem 3.2. Suppose $f \in A_{p}$ is not flowable. Then $C_{f}(G)$ is abelian, and there exist positive integers $q$ and $\delta$ with $\delta \mid q$ and $q \mid p$ and elements $\tau$ and $\omega \in C_{f}(G)$ such that

1. $C_{f}(G) / C_{f}\left(G_{1}\right)$ is cyclic of order $q$,

2. $C_{f}(G)$ is generated by $\tau$ and $f^{1 / d}$

3. $\omega$ has finite order $\delta$,

4. we have a direct product decomposition $C_{f}(G)=\langle\tau\rangle \times\langle\omega\rangle$, and finally

5. we have the relation

$$
\tau^{\frac{q}{\delta}}=\omega f^{1 / d}
$$

The formal centraliser of an $f \in G_{1}$ (other than 1) is always isomorphic to the product of a flow and a finite cyclic group. Thus $C_{f}\left(G_{1}\right)$ is isomorphic to an additive subgroup of $\mathbb{C}$. The achievement of Baker and Liverpool was to show that the only possible subgroups that can occur are $\mathbb{C}$ itself and an infinite cyclic group $\mathbb{Z} \alpha$, for some $\alpha \in \mathbb{C}$. In the latter case, $f$ has only a finite number of compositional roots. In particular, if $f$ is real-flowable, or infinitely-divisible, or lies in the image of a $\mathbb{Z}^{2}$ action, then it must be complex-flowable.

Voronin [12] used the EV data to characterise divisibility of the elements $f \in G_{1}$, i.e. the existence of composition roots. In fact, for a given $f \in G_{1}$ and $k \in \mathbb{N}$, there exists $g \in G_{1}$ with $g^{k}=f$, if and only if $\Phi=\Phi(f)$ satisfies

$$
\Phi_{j}\left(\zeta+\frac{1}{k}\right)=\Phi_{j}(\zeta)+\frac{1}{k}
$$

for $j=1, \ldots, 2 p(f)$.

In view of the Realisation Theorem, this means that generic $f \in G_{1}$ have no roots at all.

The above theorems are deep, but may be proved rather more easily than in the the original papers, by using Voronin's approach [12]. The flowable $f \in G_{1}$ are characterised as those that have EV data equivalent to $\Phi_{j}(\zeta)=\zeta+\lambda_{j}$, for constant $\lambda_{j}$, i.e. data that are translations. 


\section{Reversers}

After these preliminaries, we are ready to discuss reversibility in $G$. First, we deal with the case $m(f)=-1$. Then we proceed to prove the results stated in Section 1.3, and to provide the examples promised.

\subsection{Multiplier -1}

First, we deal with the case $m(f)=-1$. From Corollary 2.4 we deduce:

Corollary 4.1. Let $f \in G$ have $f^{\prime}(0)=-1$. Then (i) $f$ is an involution or $R_{f}(G)=R_{f^{2}}(G)$, and (ii) $f \in R(G) \Leftrightarrow f^{2} \in R(G)$.

\subsection{Proof of Theorem 1.4}

We make use of formal series arguments below. It is also possible to prove some of the results by considering separately the flowable and non-flowable germs, and using the Baker-Liverpool theory on the latter.

Let $\mathfrak{G}$ denote the group of formally-invertible series, under the operation of formal composition.

To prove Theorem 1.4, fix $p \in \mathbb{N}$, a reversible $f \in A_{p}$, and $g \in R_{f}(G)$.

Since $f \in R(G)$, then considered as a formal series, it belongs to $R(\mathfrak{G})$. Hence [10, Corollary 6] there exists a formal series $\tau \in R_{f}(\mathfrak{G})$, of order $2 p$.

Formally, $f$ is uniquely flowable [1], i.e. there exists a unique flow $\left(f^{t}\right)_{t \in \mathbb{C}}$ in $\mathfrak{G}$

with $f^{1}=f$. Also, $C_{f}(\mathfrak{G})$ is the set generated by $\tau^{2}$ and the $f^{t}, t \in \mathbb{C}$. This is well-known $[1,4,8]$, but quite concretely $f$ is formally-conjugate $[10$, Theorem 5] to

$$
\frac{z}{\left(1+z^{p}\right)^{1 / p}}
$$

and the same conjugacy takes $f^{t}(z)$ to

$$
\frac{z}{\left(1+t z^{p}\right)^{1 / p}} \text {. }
$$

For all $t \in \mathbb{C}$, the latter commutes with $z \mapsto \exp (2 \pi i / p) z$, and is reversed $z \mapsto \exp (\pi i / p) z$, and $\tau$ is obtained by conjugating the latter back.

In particular, $\tau$ reverses each $f^{t}$, for $t \in \mathbb{C}$.

Now $\tau^{-1} g \in C_{f}(\mathfrak{G})$, and hence $\tau^{-1} g=\tau^{2 r} f^{t}$ for some $r \in \mathbb{Z}$ and $t \in \mathbb{C}$, so $g=$ $\tau^{m} f^{t}$ for for an odd $m \in \mathbb{Z}$. Since $\tau^{m}$ reverses $f^{t}$, we get $g^{2}=\tau^{m} f^{t} f^{-t} \tau^{m}=\tau^{2 m}$, so the order of $g^{2}$ divides $p$, so the order of $g$ is finite, dividing $2 p$.

The order of $g$ cannot be odd (since $f$ is not involutive), and hence it is $2 s$, for some $s \mid p$. Finally, if $p / s$ were even, we would have $m(g)^{p}=1$, but a simple formal calculation shows that $g$ cannot reverse $f$ unless $m(g)^{p}=-1$. 


\subsection{Proof of Theorem 1.5}

This is immediate from Corollary 1.2(2) and Theorem 1.4.

\subsection{Proof of Theorem 1.6}

Suppose $f \in R(G)$. By Theorem 1.4, there exists $g \in R_{f}$, of order $2 s$, with $p / s$ odd. Thus there is a function $\psi \in H$ that conjugates $g$ to $\beta z$, where $\beta=m(g)$.

Then $\psi^{-1} f \psi$ is reversed by $\beta z$, and commutes with $\beta^{2} z$. Since $\beta^{2}$ is a primitive $s$-th root of unity, it follows that $\psi^{-1} f \psi$ takes the form given by equation (1.1). Since $\beta z$ reverses it,

$$
\psi^{-1} f^{-1} \psi(z)=\beta^{-1}\left(\psi^{-1} f \psi\right)(\beta z)
$$

takes the form (1.2).

This proves one direction, and the converse is obvious.

\subsection{Proof of Corollary 1.7}

It is true in any group that $f \in R(G) \Rightarrow f^{2} \in R(G)$. For the converse in our specific $G$, there are two cases: $m(f)= \pm 1$.

If $m(f)=1$, and $f^{2} \in R(G)$, then we have seen in the proof of Theorem 1.4 that each reverser of $f^{2}$ reverses each element of the formal flow $\left(f^{2}\right)^{t}$, and hence reverses $\left(f^{2}\right)^{1 / 2}=f$. (Observe that if a convergent series is formally reversed by a convergent series, then it is holomorphically reversed by it, too.)

If $m(f)=-1$, and $f^{2} \in R(G)$, then we have $f \in R(G)$ by Proposition 1.3, Part 2 .

\subsection{Example: Reversible germ, not reversible by any germ of order dividing $2^{k}$}

Fix any even $p \in \mathbb{N}$, and take $s=p$. Let $\mu \in G$ be multiplication by a primitive $s$-th root of -1 . Take $\phi \in G_{1}$ commuting with $\mu^{2}$, but not with $\mu$. (This may be done, for instance, by taking $\phi(z)=z+z^{s+1}$.) Take $g=\mu, h=\phi^{-1} \mu \phi$, and $f=g^{-1} h$. Then a calculation shows that $g^{2}=h^{2}$ has order $s$ (and hence $g$ is a reverser for $f$ of order $2 s$ ), and that $f \in A_{p}$. In case $p=2^{k+1}$, we see (by Theorem 1.4) that no element of order $2^{k}$ can reverse $f$.

Another example is provided by the function $z\left(1+z^{p}\right)^{-1 / p}$ used in the proof of Theorem 1.4, in view of Corllary 1.8. Examples of this kind may also be constructed (rather less concretely) by appealing to the Realization Theorem). However, the Realization Theorem is the best way to do the next thing: 


\subsection{Example: Non-flowable reversible germ}

Fix any $p \in \mathbb{N}$, and take $\mathrm{EV}$ data $\Phi$, where

$$
\Phi_{1}(\zeta)=\zeta+\exp (-2 \pi i \zeta), \quad \Phi_{2}(\zeta)=\zeta-\exp (2 \pi i \zeta)
$$

and $\Phi_{j+2}=\Phi_{j}$ for all $j$.

By the Realization Theorem, there is some $f \in A_{p}$ with $\mathrm{EV}$ data $\Phi(f)$ equivalent to $\Phi$. Hence this $f$ is reversible, by Proposition 1.3 , because $\left(-\Phi_{j+1}(-\zeta)\right)$ is the $\mathrm{EV}$ data for $f^{-1}$. (This is so, because the consecutive attracting and repelling petals for $f$ are, respectively, repelling and attracting for $f^{-1}$, and because $F_{j+1}$ conjugates $f^{-1}$ in the $j+1$-st petal to to $\zeta \mapsto \zeta-1$ near $\infty$, so that $-F_{j+1}(-\cdot)$ conjugates $f^{-1}$ to $\zeta \mapsto \zeta+1$, so that the $\mathrm{EV}$ recipe gives $-F_{j+1}\left(--F_{j+2}^{-1}(-\zeta)\right)=$ $-\Phi_{j+1}(-\zeta)$ as EV data for $f^{-1}$.)

But since $\Phi_{1}$ is not a translation, $f$ is not flowable.

\subsection{Example: Formally-reversible germ, not reversible in G}

Let $\Phi_{1}(\zeta)=\zeta+e^{-2 \pi i \zeta}$ and $\Phi_{2}(\zeta)=\zeta$. If $f$ realizes this EV data then $a(f)=$ $1=(p+1) / 2$ by the formula on top of page 19 of [5], and hence $\mathrm{f}$ is formally reversible, but these data do not have the symmetry required of reversible germ data.

\section{The Order of a Reverser}

Flowable reversible germs $f \in A_{p}$ are very special: they form a single conjugacy class - all are conjugate to $z /\left(1+z^{p}\right)^{1 / p}$, and all reversers for them have order dividing $2 p$. The possible orders are precisely the divisors of $2 p$ of the form $2^{k} u$, where $u \mid p$ is odd, and $2^{k}$ is the largest power of 2 dividing $2 p$.

In the nonflowable case, we can relate the possible orders for reversers to the centraliser generators $\tau, \omega$, and the natural numbers $d, q$ and $\delta$ of Theorem 3.2. The numbers $d, q$, and $\delta$ are uniquely-determined by $f$ : the $1 / d$-th power of $f$ is the smallest positive power that converges, $q$ is the index of $C_{f}\left(H_{1}\right)$ in $C_{f}(G)=C_{f}(H)$, and $\delta$ is the order of the (cyclic) torsion subgroup of $C_{f}(G)$. The germ $\omega$ may be any generator of this torsion subgroup; we may specify a unique $\omega$ by requiring that the multiplier $m(\omega)=e^{\frac{2 \pi i}{\delta}}$ (as opposed to some other primitive $\delta$-th root of unity).

Theorem 5.1. Let $p \in \mathbb{N}$, and suppose $f \in A_{p}$ is reversible but not flowable. Let $\tau, \omega$ and $d, q, \delta$ be as in Theorem 3.2. Then 
1. If $g \in R_{f}(G)$ then $g$ commutes with $\omega$, and $g$ reverses $f^{r / d}$, for each $r \in \mathbb{Z}$.

2. $\delta=q$, and $\frac{p}{q}$ is odd.

3. If we choose $\omega$ such that $m(\omega)=e^{\frac{2 \pi i}{\delta}}$, then we have

$$
\left\{g^{2}: g \in R_{f}(G)\right\}=\left\{\omega^{l}: l \text { is odd }\right\},
$$

and we always have

$$
\left\{\operatorname{ord}(g): g \in R_{f}\right\}=\{2 r \in \mathbb{N}: r \mid q \text {, and } q / r \text { is odd }\} .
$$

Proof. We abbreviate $R_{f}=R_{f}(G)$. (1) Since $g$ (and hence $g^{-1}$ ) reverse $f$ and $\omega$ commutes with $f$ we see that $g \omega g^{-1}$ commutes with $f$, has order $\delta$ and has the same multiplier as $\omega$, and so it equals $\omega$. To show the second part of 1 , it suffices to deal with the case $r=1$. Again $g f^{\frac{1}{d}} g^{-1}$ commutes with $f$ and it has multiplier 1 so $g f^{\frac{1}{d}} g^{-1}=f^{\frac{l}{d}}$ for some $l$. Raise both sides of the last equation to the power $\mathrm{d}$ to get $f^{-1}=f^{l}$ and so $l=-1$ as desired. This proves part 1 .

(2) We know that if $g \in R_{f}$ then $g^{\prime}(0)^{p}=-1, g^{2}$ commutes with $f$ and that $g$ has finite order. It follows that $g^{\prime}(0)=e^{\frac{\pi i m}{p}}$ where $m$ is odd. Since $g^{2}$ is periodic and commutes with $f$ we have $g^{\prime}(0)^{2 \delta}=1$ i.e. $e^{\frac{2 \pi i m \delta}{p}}=1$. This means that $m=\frac{p}{\delta} l$ for some integer $l$. Since $m$ is odd, so also are $\frac{p}{\delta}$ and $l$. So far we have seen that $\frac{p}{\delta}$ is odd. Now we show that $q=\delta$. Now $g \tau g^{-1} \tau^{-1}$ commutes with $f$ and has multiplier 1 so $g \tau g^{-1} \tau^{-1}=f^{\frac{n}{d}}$ for some integer $n$. If we take this last identity and raise both sides to the power q we get $g f^{\frac{\delta}{d}} g^{-1} f^{\frac{-\delta}{d}}=f^{\frac{q n}{d}}$. Now using the fact that $g$ reverses $f^{\frac{l}{d}}$ we arrive at $-2 \delta=q n$. So $-2=\frac{q}{\delta} n$ so that $\frac{q}{\delta}$ is either 1 or 2 . But $q=2 \delta$ is not consistent with the fact that $\frac{p}{\delta}$ is odd. Hence $q=\delta$.

(3) Pick any $g \in R_{f}$. We already know from Theorem 1.4 that $g$ has finite order. Since $g^{2} \in C_{f}$, it follows that $g^{2}$ belongs to the torsion subgroup, and hence is a power $\omega^{l}$. If $l$ were even, then $m(g)^{p}=1$, but a reverser of $f$ must have $m(g)^{p}=-1$. This proves that

$$
\left\{g^{2}: g \in R_{f}\right\} \subset\left\{\omega^{l}: l \text { is odd }\right\} .
$$

To see the opposite inclusion, fix $g_{0} \in R_{f}$, with $g_{0}^{2}=\omega^{l}$. Then $\omega^{j} g_{0} \in R_{f}$ whenever $j \in \mathbb{Z}$, and the square of this reverser is $\omega^{2 j} g_{0}^{2}=\omega^{l+2 j}$. Letting $j$ run through $\delta$ consecutive integers, we get each odd power of $\omega$. Thus

$$
\left\{g^{2}: g \in R_{f}\right\}=\left\{\omega^{l}: l \text { is odd }\right\} .
$$

We conclude that the possible values of ord $(g)$ are the numbers 2 ord $\left(\omega^{l}\right)$, where $l$ ranges over the odd numbers. Since $\omega$ has order $\delta=q$, the order of $\omega^{l}$ is $r=q / u$, where $u$ is the greatest common divisor of $l$ and $\delta$. Since $l$ is odd, $u$ must be odd as well. Conversely, suppose that $r$ is a divisor of $q$ and $u=q / r$ is odd. Then by the last equation there is a $g \in R_{f}$ with $g^{2}=\omega^{u}$, which obviously has order $r$. 
Corollary 5.2. If $p=2^{k} u$ where $u$ is odd, and $f \in A_{p}$ is nonflowable and reversible in $G$, then $\delta=q=2^{k} n$ where $n$ divides $u$. The largest order for a reverser of $f$ is $2 \delta$ and the smallest order is $2^{k+1}$.

Note that in the flowable case, this corollary also holds (with, additionally, $q=p$ ).

Using EV theory it can be shown that given any positive integer $\mathrm{p}$ and any divisor $q$ of $p$ such that $\frac{p}{q}$ is odd then there is a reversible $f \in A_{p}$ such that the associated $q_{f}=q$, and in fact an infinite dimensional set of inequivalent ones.

\section{References}

[1] Baker, I.N. Permutable power series and regular iteration. J. Australian Math. Soc. 2 (1962) 265-94.

[2] Baker, I.N. Fractional iteration near a fixpoint of multiplier 1. J. Australian Math. Soc. 4 (1964) 143-8.

[3] Baker, I.N. Nonembeddable functions with a fixpoint of multiplier 1. Math. Zeitschr. 99 (1967) 377-84.

[4] Liverpool, L.S.O. Fractional iteration near a fix point of multiplier 1. J. Lond. Math. Soc. (2) 9 (1975) 599-609.

[5] Ahern, Patrick and Gong, Xianghong. A complete classification for pairs of real analytic curves in the complex plane with tangential intersection. J. Dynamical and Control Systems. 11 (2005) 1-71.

[6] Ilyashenko, Yu.S. Nonlinear Stokes phenomena. In: Nonlinear Stokes Phenomena. Adv. Sov. Math. 14 American Math. Soc., Providence, RI. (1993) $1-55$.

[7] Kuczma, M., Choczewski, B. and Ger, R. Iterative Functional Equations. Cambridge University Press. 1990.

[8] Lubin, Jonathan. Nonarchimedean dynamical systems. Compositio Math. 94 (1994) 321-46.

[9] Malgrange, Bernard. Travaux dÉcalle et de Martinet-Ramis sur les systemes dynamiques. Seminaire Bourbaki 34e année 1981/82. Asterisque no. 582 (1981) 59-73.

[10] O'Farrell, Anthony G. Composition of involutive power series, and reversible series. Computational Methods in Function Theory. 8 (2008) 173-93. 
[11] Szekeres, G. Fractional iteration of entire and rational functions. J. Australian Math. Soc. 4 (1964) 129-42.

[12] Voronin, S.M. Analytic classification of germs of conformal maps $(\mathbb{C}, 0) \rightarrow$ $(\mathbb{C}, 0)$ with linear part the identity. Funct. Anal. Appl. 15, no. 1, (1981) 1-17.

[13] Voronin, S.M. Analytic classification of pairs of involutions and its applications. Funct. Anal. Appl. 16, no. 2, (1982) 94-100 